\title{
ANÁLISE DA SUSTENTABILIDADE HIDROAMBIENTAL DE MUNICÍPIOS DA REGIÃO DA SUB-BACIA DO ALTO PIRANHAS, PB
}

\author{
J. R. M. CARVALHO*, G. A. CÂNDIDO, W. F. CURI e E. K. M. DE ARAÚJO ${ }^{1}$ \\ Universidade Federal de Campina Grande \\ profribamar@gmail.com*
}

Artigo submetido em janeiro/2015 e aceito em outubro/2015

DOI: $10.15628 /$ holos.2015.2093

\section{RESUMO}

A problemática dos recursos hídricos se configura como uma questão crucial para a superação dos obstáculos do desenvolvimento. Nesse sentido, e em meio a uma diversidade de sistemas de indicadores hidroambientais desenvolvidos para mensurar e subsidiar o processo de decisão no âmbito da formulação de políticas públicas relacionadas a gestão da água, foi elaborada essa proposta, composta por 46 indicadores com o objetivo de avaliar o nível de sustentabilidade hidroambiental dos municípios localizados na região da sub-bacia hidrográfica do Alto Piranhas, PB. Para tanto, fez-se uso da pesquisa exploratória e documental e foram adotados procedimentos da estatística descritiva. Os resultados obtidos apontam que os municípios com melhores performances hidroambientais, obtidas a partir das variáveis selecionadas do modelo, foram: Cajazeiras, Sousa, Cachoeira dos Índios, Uiraúna, Santa Cruz, Santa Helena e Lastro. Já os municípios com piores performances foram: Poço Dantas, Santarém, Vieirópolis e Bernardino Batista. Entende-se, portanto, que a ampliação do número de indicadores e a aplicação em outras sub-bacias ou bacias hidrográficas do estado paraibano pode subsidiar a definição de melhores estratégias de gestão pública local e regional em relação à sustentabilidade hidroambiental.

PALAVRAS-CHAVE: Sustentabilidade hidroambiental. Gestão Pública. Sertão Paraibano.

\section{ANALYSIS OF SUSTAINABILITY HYDROENVINRONMENTAL IN REGION OF HYDROGRAPHIC BASIN OF ALTO PIRANHAS, PB}

\begin{abstract}
The issue of water resources is configured as a crucial issue for overcoming the obstacles of development. In this sense, and in the midst of a diversity of hydroenvironmental indicator systems designed to measure and support the decision making process within the formulation of public policies related to water management, it was drafted this proposal, composed of 46 indicators to evaluate the level of hydroenvironmental sustainability of municipalities in the watershed of the region of Alto Piranhas, PB. Therefore, it made use of exploratory and documentary research. Descriptive statistics procedures were adopted. The
\end{abstract}

results suggest that municipalities with hydro-best performances, obtained from the model selected variables were: Cajazeiras, Sousa, Cachoeira dos Indios, Uiraúna, Santa Cruz, Santa Helena and Lastro. The municipalities with the worst performances were: Poço Dantas, Santarém, Vieirópolis, and Bernardino Batista. It is understood that increasing the number of indicators and the application in other sub-basins or river basins of the Paraíba state can support the definition of the best strategies for local and regional public administration in relation to hydro-environmental sustainability.

KEYWORDS: hydro-environmental sustainability. Public Administration. Backlands of Paraiba. 


\section{INTRODUÇÃO}

O interesse e a preocupação sobre questões ambientais assumiram grande importância no debate científico e político, de maneira mais intensificada, a partir das décadas de 60 e 70 do século $\mathrm{XX}$, quando surgiram movimentos ambientalistas, regulamentações, organizações não governamentais, dentre outros, impulsionados, principalmente, pelo contexto em que se encontram os atuais sistemas convencionais de gestão de recursos naturais de uso compartilhado.

Dados sobre o crescimento da população mundial assinalam hoje mais de seis bilhões de seres humanos, o que poderá chegar a oito bilhões em 2025. Nesse cenário, o desenvolvimento tecnológico com a respectiva geração de resíduos em diferentes graus de toxicidade, o consumo descontrolado da água, a crise climática, o crescimento das cidades, são exemplos reais que fizeram a água migrar da condição "abundante e limpa" para "escassa e poluída" em muitos países. No Brasil estão localizadas algumas das maiores bacias hidrográficas do mundo e, não por acaso, cerca de $90 \%$ de energia elétrica produzida tem origem hidráulica. Essa enorme disponibilidade levou a população brasileira a mascarar o desperdício e o uso predatório com a desculpa da abundância, numa tentativa de justificar a ideia de que a água é um bem ilimitado (MARANHÃO, 2007).

Segundo Policarpo e Santos (2008), diante de uma crise socioambiental que deveria ser pensada em sua dimensão complexa e sistêmica, os sistemas convencionais de gestão de recursos naturais de uso compartilhado, como é o caso da água, não consideram o potencial contido nos diferentes sistemas de autoridade construídos e administrados no nível local, nem os diferentes tipos e interesses de atores sociais, chaves para a gestão.

Particularmente, no âmbito da gestão de águas no Brasil, observa-se que o seu desenvolvimento ainda se deu de forma fragmentada e centralizada. De acordo com Abers e Jorge (2005), a gestão das águas era fragmentada, e ainda é, em função de cada setor (energia elétrica, agricultura irrigada, saneamento etc.) realizar seu próprio planejamento, e centralizada pelo fato de que o governo no âmbito estadual e federal, ainda define a política de gestão das águas sem que houvesse a participação dos governos municipais, dos usuários da água e da sociedade civil [grifos dos autores].

Pelo exposto, entende-se que uma análise do estado atual das questões hídricas em contextos geográficos de municípios, microbacias hidrográficas considerando dimensões / categorias relacionadas ao desempenho do sistema (desenvolvimento humano; econômico; abastecimento humano; pressões da irrigação, pecuária, abastecimento rural, aquicultura e lazer; coleta de esgoto; atendimento de coleta de lixo; e estado qualitativo da água) pode permitir a inferência sobre as implicações diretas, em escala espacial e temporal, capaz de contribuir para o monitoramento da gestão dos recursos hídricos.

Existe uma diversidade de sistemas de indicadores de sustentabilidade hidroambientais (HE et al., 2000; POMPERMAYER, PAULA JÚNIOR, CORDEIRO NETTO, 2007; LOPES et al. 2009; ARAÚJO, RIBEIRO, VIEIRA, 2012; CARVALHO, CURI, 2013). No entanto para os fins da pesquisa realizada, tomou-se como referência, os estudos desenvolvidos por Guimarães (2008), Martins e Cândido (2008), Vieira e Studart (2009) e Magalhães Júnior (2010), que exploram os indicadores de 
sustentabilidade dentro de abordagens mais relacionadas com características dos recursos hídricos do Semiárido brasileiro.

A partir destas considerações, o objetivo do artigo é avaliar o nível de sustentabilidade hidroambiental dos municípios localizados na região da sub-bacia hidrográfica do Alto Piranhas, PB. Em termos metodológicos, trata-se de pesquisa descritiva e exploratória, utilizando a parametrização dos indicadores utilizados, dentro de critérios e parâmetros de avaliação prédefinidos.

O artigo apresenta além dessa seção de introdução, o suporte teórico do estudo, constituído da apresentação dos conceitos da literatura existentes acerca do tema, o método de pesquisa que norteará o estudo, a análise e discussão dos resultados, e, por fim, as considerações finais.

\section{REFERENCIAL TEÓRICO}

\subsection{INDICADORES PARA A SUSTENTABILIDADE HIDROAMBIENTAL}

O maior desafio, quando se trata de discutir a questão da sustentabilidade, é o de compatibilizar o crescimento econômico com a preservação ambiental e justiça social. Para isso, o gerenciamento do conhecimento no uso dos indicadores de sustentabilidade pode ser um instrumento adequado para tais evidências, principalmente porque nestes sistemas de indicadores estão contidas todas as informações pertinentes à situação econômica, social e ambiental de um espaço geográfico em um determinado período (LIRA, 2008).

Os indicadores de sustentabilidade aparecem como ferramentas capazes de subsidiar o monitoramento da operacionalização do desenvolvimento sustentável, tendo como função principal a revelação de informações sobre o estado das diversas dimensões (ambientais, econômicas, socioeconômicas, culturais, institucionais, etc.) que compõem o desenvolvimento sustentável do sistema na sociedade.

Os indicadores de sustentabilidade são utilizados como informações relevantes em diversos estudos nacionais e internacionais, facilitando a compreensão dos fenômenos complexos, e atua como base para análise do desenvolvimento que abrange diversas dimensões (fatores econômicos, sociais, culturais, geográficos e ambientais), uma vez que permite verificar os impactos das ações humanas no ecossistema (SILVA; CORREIA; CÂNDIDO, 2010).

As definições mais comuns de indicadores e a terminologia associada a essa área são particularmente confusas, tanto em relação à definição de indicadores quanto a outros conceitos associados como: índice, meta e padrão. No entanto, entende-se, que o presente estudo busca agregar e quantificar informações de um modo que sua significância fique mais aparente e transparente, simplificando informações sobre tais fenômenos tentando melhorar com isso o processo de comunicação e de decisão.

De acordo com Van Bellen (2006), os indicadores podem ser quantitativos ou qualitativos. Os mais adequados para a avaliação de experiências de desenvolvimento sustentável deveriam ser mais qualitativos, em função das limitações explícitas e implícitas que existem em relação a indicadores simplesmente numéricos, muito embora, em alguns casos, avaliações qualitativas podem ser transformadas numa notação quantitativa (VAN BELLEN, 2006). 
Como se observa, a utilização de indicadores é uma maneira intuitiva de monitorar questões sociais, econômicas e ambientais, visto que dependendo da forma como são estruturados, analisados, podem evidenciar elementos importantes relacionados à forma como a sociedade se relaciona com a natureza e o seu habitat.

Todavia, alguns cuidados devem ser tomados quando da escolha de indicadores, uma vez que diferentes tipos de indicadores podem, em determinadas situações, ser relevantes em diferentes escalas e, também, podem perder o seu sentido quando utilizados sem o devido cuidado em escalas não apropriadas. Outro aspecto destacado na discussão dos indicadores relacionados ao desenvolvimento sustentável consiste na dimensão do tempo, podendo ser escalares (número simples gerado da agregação de dois ou mais valores) e vetoriais (geração de uma variável com magnitude e direção de característica bidirecional - tendência de futuro com viés holístico), além dos valores existentes na sua escolha, que podem ser explícitos (tomados conscientemente e que compreendem uma parte fundamental no processo de criação de indicadores) ou implícitos decorrem de aspectos que não são facilmente observáveis e que são, na sua maioria, inconscientes e relacionados a características pessoais e de uma determinada sociedade (VAN BELLEN, 2006).

Especificamente, em relação ao conceito de indicador hidroambiental argumenta-se como sendo um indicador / índice que apresenta uma conotação de correlação entres vários aspectos sociais, econômicos e ambientais e sua suposta relação com o recurso natural água, ou seja, corresponde a uma análise multidisciplinar tratando de vários aspectos de inter-relacionamento entre parâmetros hídricos e ambientais tendo como base alguns critérios importantes como: desenvolvimento humano, desenvolvimento econômico, abastecimento humano, pressões da irrigação, pecuária, abastecimento rural, aquicultura e lazer, coleta de esgoto, atendimento de coleta de lixo e estado qualitativo da água.

\subsection{ESTUDOS CORRELATOS DA TEMÁTICA DO ESTUDO}

A literatura nacional e internacional evidencia algumas experiências em relação à utilização de modelos de indicadores de sustentabilidade como maneira de subsidiar o gerenciamento das bacias hidrográficas. A seguir estão evidenciados os estudos que nortearam esta pesquisa e que utilizaram indicadores hidroambientais.

He et al. (2000) sugeriram uma estrutura para desenvolver e testar um conjunto de indicadores hidrológicos e biológicos que refletem a condição de uma bacia hidrográfica. Demonstraram que o uso de indicadores hidroambientais no processo de planejamento de bacias hidrográficas requer interações sistemáticas, tais como reuniões, pesquisas e discussões com as partes interessadas, ou seja, os atores sociais envolvidos.

Magalhães Júnior, Cordeiro Netto e Nascimento (2003) apresentam a síntese dos resultados de um painel Delphi aplicado no país, envolvendo os indicadores mais valorizados e as tendências de pensamento quanto aos principais meios de ação na gestão das águas no país.

Curi, Curi e Oliveira (2004) utilizaram indicadores para avaliar a sustentabilidade hídrica através da programação linear e da análise de sensibilidade em um perímetro irrigado localizado no Semiárido paraibano.

Laura (2004) desenvolveu um método de modelagem de sistema de indicadores para avaliar a sustentabilidade do sistema dos recursos hídricos, propiciando a participação dos atores 
sociais e visando ter maior conhecimento do problema e legitimidade de gestão dos recursos hídricos numa bacia hidrográfica no estado do Paraná. Esse autor elenca algumas formas interessantes para se construir índices e indicadores de gestão hídrica, demonstrando a relevância de considerar vários aspectos e parâmetros dentro desse contexto.

Por outro lado, Pompermayer, Paula Júnior e Cordeiro Netto (2007) propuseram o uso de indicadores de sustentabilidade ambiental, associado às técnicas de análise multicritério, como instrumento de auxílio à gestão de recursos hídricos. A proposta de indicadores selecionada e o método multicritério utilizado (Electre III) demonstraram-se bastante adequados a região das Bacias dos Rios Piracicaba, Capivari e Jundiaí no estado de São Paulo.

Guimarães (2008) desenvolveu uma proposta metodológica para sistema de indicadores de desenvolvimento sustentável para bacias hidrográficas brasileiras. Nessa metodologia, a autora propõe um índice agregado com 8 indicadores relacionados à dimensão social, 20 relacionados à dimensão ambiental, 8 de natureza econômica e 4 relacionados à dimensão institucional.

Vieira e Studart (2009) propuseram um modelo de Índice de Sustentabilidade Hidroambiental (ISHA) para Ambientes Serranos no Semiárido do Estado do Ceará, como é o caso da Área de Proteção Ambiental (APA), do Maciço Baturité. O modelo estrutural baseou-se na abordagem Pressão-Estado-Resposta da OECD (1998). O ISHA mostra a posição relativa e a posição absoluta de cada município dos seguintes índices: Índice Hídrico (8 indicadores), Índice Físico (4 relacionados), Índice Biótico (4 relacionados) e Índice Antrópico (12 indicadores).

Magalhães Júnior (2010) aponta uma série indicadores ambientais, potencialmente úteis à gestão da água no Brasil, envolvendo sete indicadores relacionados a dimensão de cobertura vegetal, 4 indicadores relacionados ao estado qualitativo dos estoques hídricos, 14 indicadores relacionados a dinâmica fluvial e riscos de não atendimento às demandas, 21 indicadores relacionados ao estado qualitativo da água, 13 indicadores relacionados às pressões ou impactos sobre as águas e ambientes aquáticos, 15 indicadores acerca das pressões de disponibilidade hídrica (redução dos estoques hídricos), cinco indicadores a relacionados às pressões sobre a qualidade das águas (pressões agrícolas e industriais), 25 indicadores acerca de indicadores de respostas/desempenho do sistema de gestão da água, e 11 indicadores de desempenho do sistema de gestão quanto aos aspectos legais, institucionais, financeiros e de fiscalização.

Kodikara et al. (2010) avaliaram as regras de funcionamento dos sistemas de reservatórios de abastecimento de água urbana, considerando as seguintes perspectivas dos seguintes atores sociais: gestores de recursos, usuários da água e meio ambiente. Além disso, os autores utilizaram um conjunto de variáveis específicas para identificar pesos e graus de importância destas variáveis, utilizando técnicas de análise multivariada.

Mutikanga et al. (2011) utilizaram o método PROMETHEE II para reduzir perdas na distribuição de água na cidade de Kampala, Uganda. O estudo se baseia nas preferências dos tomadores de decisão e adota critérios de avaliação caracterizados por aspectos econômicofinanceiros, ambiental, de saúde pública, impactos técnicos e sociais.

Roozbahani et al. (2012) realizaram um estudo de caso no sistema de abastecimento de água em Melbourne, para avaliar um conjunto de regras de funcionamento em relação a oito critérios avaliados. Os resultados encontrados se mostraram mais alinhados a realidade local. 
Nos estudos supramencionados, fica evidente a importância da aplicação em áreas distintas relacionadas à gestão dos recursos hídricos, mostrando-se como o processo de decisão pode ser beneficiado. Afinal, atuar em um cenário aonde decidir sobre qual opção escolher entre vários critérios conflitantes e diferentes interesses das partes é uma tarefa desafiadora.

Como se observa existe a necessidade de entender melhor a maneira como tais indicadores ou indicadores foram propostos no intuito de definir melhores formas de calcular e analisar o contexto gestão hidroambiental de municípios, posto ser uma das características implícitas existentes quando da elaboração de metodologias ou ferramentas que utilizam indicadores / índices.

\section{PROCEDIMENTOS METODOLÓGICOS}

A metodologia utilizada neste estudo consistiu em uma pesquisa exploratória, e documental, a partir do uso da estatística descritiva. Foram escolhidos alguns indicadores hidroambientais, de acordo com a disponibilidade de dados (censos, institutos, agências, dentre outros), a fim de avaliar a sustentabilidade hidroambiental dos municípios localizados na região da sub-bacia hidrográfica do Alto Curso do Rio Piranhas. Os estudos que embasaram a escolha dos indicadores foram os trabalhos de Magalhães Júnior (2010), Vieira e Studart (2009), Guimarães (2008), Martins \& Cândido (2008).

Para este trabalho, foram levantados dados cujas diferenças temporais fossem as mínimas possíveis. Esses dados variaram entre os anos de 2002 a 2010. As fontes e os respectivos anos encontram-se no quadro 1.

Para a definição e representação gráfica do índice de sustentabilidade hidroambiental foi adotada a metodologia proposta por Martins e Cândido (2008).

Observou-se que as variáveis estudadas apresentam diferentes unidades de medida, fazendo com que fossem utilizadas unidades de agregação a partir das relações positivas e negativas de cada uma delas. A sequência adotada seguiu o entendimento de Sépulveda (2005), Waquil et al. (2007), Cândido e Martins (2008).

Essa estratégia adotada proporciona o ajuste de valores observados das variáveis às escalas, cujo valor mínimo é zero e valor máximo é igual um. Ou seja, cria condições para a agregação nas dimensões/categorias, e, consequentemente, a estimação de um índice, que no caso do estudo foi denominado de Índice de Sustentabilidade Hidroambiental para Municípios (ISHM).

De acordo com as propostas metodológicas apresentada por Sepúlveda (2005), Waquil et al. (2007), Martins e Cândido (2008), quando as variáveis são escolhidas, deve-se definir o tipo de relação que cada uma delas tem com a temática que se pretende investigar, no caso do estudo com a gestão da água. Nesse sentido, para cada variável é necessário identificar se ela mede uma situação em que, ao aumentar seu valor, favorece ou desfavorece o processo de desenvolvimento, no caso do estudo de sustentabilidade hidroambiental. Assim, existe uma relação positiva se um aumento no valor da variável resulta em melhoria do sistema; em contrapartida, a relação é negativa se um aumento no valor da variável resulta em piora do sistema. A operacionalização metodológica foi feita seguindo as relações das seguintes fórmulas ou equações: 


\section{Relação Positiva:}

$$
I=\frac{x-m}{M-m}
$$

\section{Relação Negativa:}

$$
I=\frac{M-x}{M-m}
$$

Onde:

I = índice calculado referente a cada variável, para cada território analisado;

$\mathrm{x}=$ valor observado de cada variável em cada território analisado;

$\mathrm{m}=$ valor mínimo considerado;

$\mathrm{M}=$ valor máximo considerado.

A definição do sinal, indicando se a relação é positiva ou negativa, foi feita a partir da intuição dos autores. Segundo Waquil et al. (2007) "na maior parte dos casos, a definição é clara e não carece de maiores explicações." Por exemplo, a variável precipitação média dos municípios têm uma relação positiva (na região semiárida), vez que, quanto maior a precipitação maiores serão, as melhorias do sistema, indicando relações positivas; por outro lado, quanto maior o percentual da população que não tem instalações sanitárias, pior para o sistema, indicando relações negativas.

Os indicadores escolhidos (46 no total) e as respectivas categorias/dimensões foram distribuídos da seguinte forma (quadro 1):

$\checkmark$ Desempenho do sistema quanto ao desenvolvimento humano - 8 indicadores;

$\checkmark$ Desenvolvimento do sistema quanto aos indicadores econômicos -5 indicadores;

$\checkmark$ Desempenho do sistema quanto ao abastecimento humano - 8 indicadores;

$\checkmark$ Desempenho do sistema quanto às pressões da irrigação, pecuária, abastecimento rural, aquicultura e lazer - 10 indicadores;

$\checkmark$ Desempenho do sistema quanto à coleta de esgotos -6 indicadores;

\begin{tabular}{|c|c|c|c|}
\hline $\begin{array}{l}\text { CATEGORIA/ } \\
\text { DIMENSÃO }\end{array}$ & INDICADOR & FONTE & $\begin{array}{l}\text { TIPO DE RELAÇÃO } \\
\text { DO INDICADOR }\end{array}$ \\
\hline \multirow{7}{*}{$\begin{array}{l}\text { DESEMPENHO DO } \\
\text { SISTEMA QUANTO AO } \\
\text { DESENVOLVIMENTO } \\
\text { HUMANO }\end{array}$} & Taxa de Alfabetização & IDEME-PB (2008) & Positiva \\
\hline & Taxa de Mortalidade Infantil & IDEME-PB (2008) & Negativa \\
\hline & $\begin{array}{c}\text { IDH-M (Índice de } \\
\text { Desenvolvimento Humano } \\
\text { Municipal) }\end{array}$ & IDEME-PB (2008) & Positiva \\
\hline & Expectativa de Vida ao Nascer & IDEME-PB (2008) & Positiva \\
\hline & $\begin{array}{l}\text { Quantidade de estabelecimentos } \\
\text { de saúde }\end{array}$ & IBGE (2009) & Positiva \\
\hline & $\begin{array}{c}\text { Taxa de hospitalização por } \\
\text { desidratação em menores de } 5 \\
\text { anos }\end{array}$ & SIAB-DATASUS (2009) & Negativa \\
\hline & $\begin{array}{l}\text { Taxa de mortalidade infantil por } \\
\text { diarreia (por } 1.000 \text { nascidos vivos) }\end{array}$ & SIAB-DATASUS (2009) & Negativa \\
\hline
\end{tabular}

$\checkmark$ Desempenho do sistema quanto ao atendimento da coleta de lixo - 4 indicadores; e

$\checkmark$ Desempenho do sistema quanto ao estado qualitativo da água -5 indicadores

Quadro 1 - Indicadores do modelo de avaliação hidroambiental. 


\begin{tabular}{|c|c|c|c|}
\hline & $\begin{array}{l}\text { População coberta pelo Programa } \\
\text { de Saúde da Família (PSF) }\end{array}$ & SIAB-DATASUS (2009) & Positiva \\
\hline \multirow{5}{*}{$\begin{array}{l}\text { DESEMPENHO DO } \\
\text { SISTEMA QUANTO AOS } \\
\text { INDICADORES } \\
\text { ECONÔMICOS }\end{array}$} & PIB per capita em reais & IBGE (2008) & Positiva \\
\hline & $\begin{array}{l}\text { Receitas de impostos e } \\
\text { transferências de recursos }\end{array}$ & DATASUS (2009) & Positiva \\
\hline & Despesa total com saúde & SIOPS-DATASUS (2010) & Positiva \\
\hline & $\begin{array}{c}\text { Despesa total com saúde por } \\
\text { habitante }\end{array}$ & SIOPS-DATASUS (2010) & Positiva \\
\hline & Transferência SUS por habitante & SIOPS-DATASUS (2010) & Positiva \\
\hline \multirow{8}{*}{$\begin{array}{l}\text { DESEMPENHO DO } \\
\text { SISTEMA QUANTO AO } \\
\text { ABASTECIMENTO } \\
\text { HUMANO }\end{array}$} & Densidade demográfica & IBGE (2010) & Positiva \\
\hline & População total & IBGE (2010) & Positiva \\
\hline & Precipitação média anual & AESA (2010) & Positiva \\
\hline & Consumo médio per capita & IDSM (2008) & Negativa \\
\hline & Outorga p/abast. hum. $\mathrm{m}^{3} / \mathrm{h}$ & AESA (2010) & Negativa \\
\hline & $\%$ abastecimento por rede geral & DATASUS, IBGE (2002) & Negativa \\
\hline & $\begin{array}{l}\% \text { de abastecimento por poço } \\
\text { nascente }\end{array}$ & DATASUS, IBGE (2002) & Negativa \\
\hline & $\begin{array}{l}\text { \% Outra forma de abastecimento } \\
\text { de água }\end{array}$ & DATASUS, IBGE (2002) & Negativa \\
\hline \multirow{10}{*}{$\begin{array}{c}\text { DESEMPENHO DO } \\
\text { SISTEMA QUANTO ÀS } \\
\text { PRESSÕES DA IRRIGAÇÃO, } \\
\text { PECUÁRIA, } \\
\text { ABASTECIMENTO RURAL, } \\
\text { AQUICULTURA E LAZER }\end{array}$} & Outorga $\mathrm{p} /$ Irrigação $\mathrm{m}^{3} / \mathrm{h}$ & AESA (2010) & Negativa \\
\hline & Outorga p/abast.rural m3/h & AESA (2010) & Negativa \\
\hline & Outorga $\mathrm{p} /$ lazer & AESA (2010) & Negativa \\
\hline & Outorga $\mathrm{p} /$ aquicultura & AESA (2010) & Negativa \\
\hline & Número de bovinos & IBGE (2009) & Negativa \\
\hline & Número de equinos & IBGE (2009) & Negativa \\
\hline & Número de assininos & IBGE (2009) & Negativa \\
\hline & Números de muares & IBGE (2009) & Negativa \\
\hline & Número de suínos & IBGE (2009) & Negativa \\
\hline & Número de caprinos & IBGE (2009) & Negativa \\
\hline \multirow{6}{*}{$\begin{array}{l}\text { DESEMPENHO DO } \\
\text { SISTEMA QUANTO A } \\
\text { COLETA DE ESGOTOS }\end{array}$} & $\%$ rede sanitária via esgoto & DATASUS, IBGE (2002) & Positiva \\
\hline & $\%$ rede sanitária via fossa séptica & DATASUS, IBGE (2002) & Negativa \\
\hline & $\begin{array}{l}\text { \% rede sanitária via fossa } \\
\text { rudimentar }\end{array}$ & DATASUS, IBGE (2002) & Negativa \\
\hline & \% rede sanitária via vala & DATASUS, IBGE (2002) & Negativa \\
\hline & $\%$ rede sanitária via rio ou lago & DATASUS, IBGE (2002) & Negativa \\
\hline & $\begin{array}{l}\text { \% que não dispõe de instalação } \\
\text { sanitária }\end{array}$ & DATASUS, IBGE (2002) & Negativa \\
\hline \multirow{4}{*}{$\begin{array}{l}\text { DESEMPENHO DO } \\
\text { SISTEMA QUANTO AO } \\
\text { ATENDIMENTO DE } \\
\text { COLETA DE LIXO }\end{array}$} & \% de lixo coletado & DATASUS, IBGE (2002) & Positiva \\
\hline & \% lixo queimado & DATASUS, IBGE (2002) & Negativa \\
\hline & $\%$ lixo enterrado & DATASUS, IBGE (2002) & Negativa \\
\hline & $\%$ lixo jogado & DATASUS, IBGE (2002) & Negativa \\
\hline \multirow{5}{*}{$\begin{array}{c}\text { DESEMPENHO DO } \\
\text { SISTEMA QUANTO AO } \\
\text { ESTADO QUALITATIVO DA } \\
\text { ÁGUA }\end{array}$} & Turbidez fora do padrão & SNIS (2008) & Negativa \\
\hline & Cloro residual fora do padrão & SNIS (2008) & Negativa \\
\hline & Turbidez & SNIS (2008) & Negativa \\
\hline & Coliformes totais & SNIS (2008) & Negativa \\
\hline & Coliformes totais fora do padrão & SNIS (2008) & Negativa \\
\hline
\end{tabular}

Fonte: Elaboração própria (2011).

O cálculo de cada indicador foi realizado utilizando os parâmetros expostos por Martins e Cândido (2008) para cada localidade, os quais foram adaptados para a determinação da sustentabilidade hidroambiental. Foi realizada a agregação desses indicadores por dimensão através da média aritmética, chegando-se ao ISHM dos municípios que estão sendo avaliados. O 
ISHM da sub-bacia foi calculado através da média aritmética dos ISHM das categorias/dimensões dos municípios. O quadro abaixo mostra a classificação e representação dos índices (variação 0 e 1):

Quadro 01 - Classificação e representação dos índices em níveis de sustentabilidade hidroambiental

\begin{tabular}{|c|c|c|}
\hline Índice (0-1) & Coloração & Performance \\
\hline $0,7501-1,000$ & & Ideal \\
\hline $0,5001-0,7500$ & & Aceitável \\
\hline $0,2501-0,5000$ & & Alerta \\
\hline $0,0000-0,2500$ & & Crítica \\
\hline \multicolumn{2}{|c|}{ Fonte: Adaptado para o estudo conforme Martins e Cândido (2008). }
\end{tabular}

Os índices de sustentabilidade com valores entre 0,0000 e 0,2500 são caracterizados pela performance que revela um estado crítico de sustentabilidade; os índices com valores entre 0,2501 e 0,5000 foram representados pela performance que revela um estado ou situação de alerta; os índices com valores entre 0,5001 e 0,7000 foram representados pela performance que revela um estado ou situação aceitável; e por último, os índices com valores entre 0,7001 e 1,0000 foram representados pela performance que revela estado ou situação ideal de sustentabilidade (MARTINS; CÂNDIDO,2008).

A sub-bacia utilizada no estudo faz parte da bacia hidrográfica do Rio Piranhas, situada no estado da Paraíba. Nessa sub-bacia estão localizados 16 municípios: Bernardino Batista, Cachoeira dos Índios, Cajazeiras, Lastro, Marizópolis, Poço Dantas, Poço José de Moura, Santa Cruz, Santa Helena, Santarém, São Francisco, São João do Rio do Peixe, Sousa, Triunfo, Uiraúna e Vieirópolis.
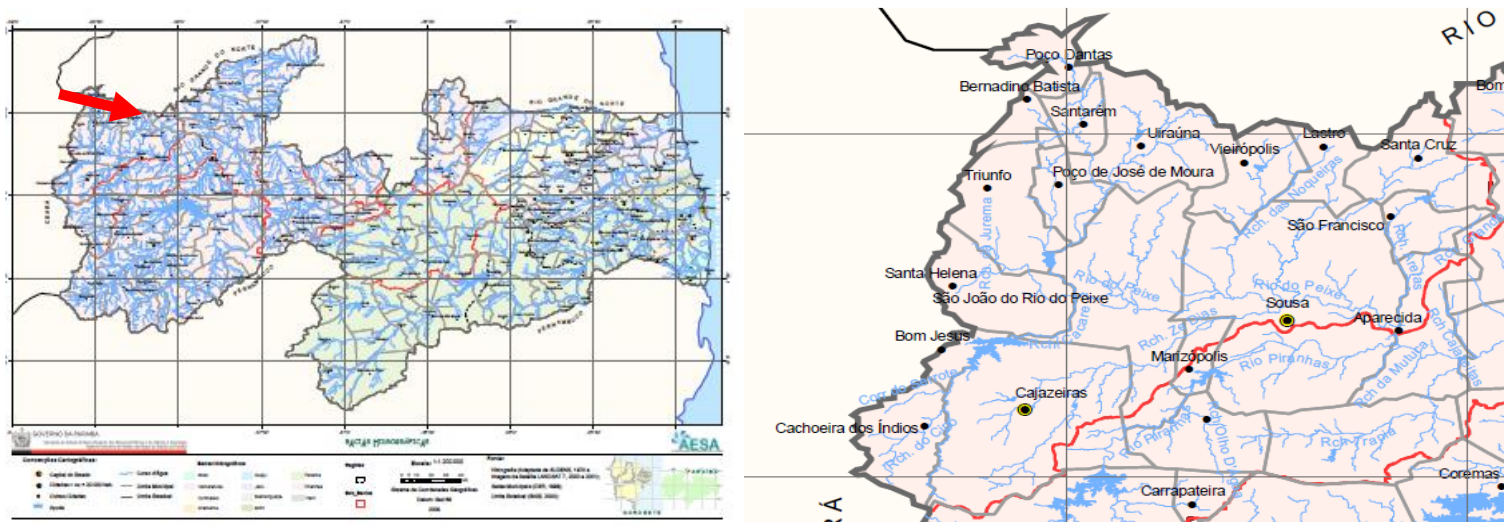

Figura 1 - Localização da área de estudo (Sub-bacia do Rio Piranhas, PB) Fonte: AESA, 2011.

A seguir estão evidenciados os resultados relacionados à caracterização dos indicadores de cada uma das dimensões analisadas e consideradas no estudo.

\section{APRESENTAÇÃO E DISCUSSÃO DOS RESULTADOS}

Após a ponderação dos indicadores relacionados ao desempenho do sistema quanto ao desenvolvimento humano, apresentados no Quadro 1, de cada município e após as análises positivas e negativas de cada um deles foi possível chegar aos resultados que estão evidenciados na Tabela 1. 
Observa-se que dos dezesseis municípios estudados, sete cidades apresentaram uma situação de alerta: Bernardino Batista $(0,49)$, Poço Dantas $(0,26)$, Poço José de Moura $(0,39)$, Santa Cruz $(0,46)$, Santarém $(0,50)$, São João do Rio do Peixe $(0,48)$ e Vieirópolis $(0,48)$. Outros sete municípios apresentaram performance aceitável: Cachoeira dos Índios $(0,53)$, Lastro $(0,54)$, Marizópolis $(0,54)$, Santa Helena $(0,61)$, São Francisco $(0,64)$, Triunfo $(0,52)$ e Uiraúna $(0,52)$. Dois municípios apresentaram performance ideal: Cajazeiras $(0,82)$ e Sousa $(0,77)$. Verifica-se que dentre os 16 municípios estudados, nenhum deles apresentou situação crítica, ou seja, entraves substanciais em relação ao desenvolvimento humano e consequentemente a situação hidroambiental local, posto que se considera a água como um vetor que contribui para o desenvolvimento humano.

Observa-se que a média geral dos municípios localizados na sub-bacia é aceitável $(0,53)$ na ótica do desenvolvimento humano. Todavia, fica evidente a necessidade de melhorar essa situação em busca da situação ideal de modo que possa contribuir satisfatoriamente para esse contexto geográfico.

Tabela 1 - Desempenho dos Municípios quanto ao Desenvolvimento Humano

\begin{tabular}{lcc}
\hline Municípios & Média do & \\
\hline Bunicípio & Performance \\
\hline Cachoeira dos Índios & 0,49 & Alerta \\
Cajazeiras & 0,53 & Aceitável \\
Lastro & 0,82 & Ideal \\
Marizópolis & 0,54 & Aceitável \\
Poço Dantas & 0,54 & Aceitável \\
Poço de José de Moura & 0,26 & Alerta \\
Santa Cruz & 0,39 & Alerta \\
Santa Helena & 0,46 & Alerta \\
Santarém & 0,61 & Aceitável \\
São Francisco & 0,50 & Alerta \\
São João do Rio do Peixe & 0,64 & Aceitável \\
Sousa & 0,48 & Alerta \\
Triunfo & 0,77 & Ideal \\
Uiraúna & 0,52 & Aceitável \\
Vieirópolis & 0,52 & Aceitável \\
\hline Média dos Municípios da Sub-bacia & 0,48 & Alerta \\
\hline
\end{tabular}

Fonte: Elaboração própria a partir dos dados da pesquisa.

Prosseguindo na análise, passa-se ao desempenho dos municípios quanto aos indicadores econômicos, segundo os parâmetros mostrados no Quadro 1, caracterizada na tabela 2. Os resultados mostram que apenas o município de Sousa apresentou uma situação ideal $(1,00)$, quando comparado aos demais, seguido de Cajazeiras $(0,62)$ com situação aceitável. A grande maioria dos municípios apresentou situação crítica $(87,5 \%)$. Talvez uma das causas desse rendimento crítico esteja relacionada à grande dependência financeira que esses municípios detêm em relação a transferências intergovernamentais, ou seja, os entraves financeiros são sanados apenas com os recursos oriundos do governo federal, o que demonstra que os anseios da sociedade podem não ser atingidos com os atuais patamares de recursos. 
Talvez esse cenário se apresente desta forma nessas localidades devido à ausência ou pouca adoção de estratégias públicas voltadas para a geração de renda a partir das potencialidades locais, denotando uma falta de política sustentável em relação a essa categoria. Por conta desse cenário, a média geral da categoria dos municípios da sub-bacia apresentou um nível crítico, que certamente influencia na adoção de práticas e iniciativas relacionadas aos recursos hidroambientais, afinal entende-se que, quanto maior for a geração de renda local, maior tende a ser o nível de instrução, a independência financeira de recursos da União, culminando na melhoria da gestão e na qualidade de vida da população.

\begin{tabular}{lcc} 
Tabela 2 - Desempenho do Sistema quanto aos Indicadores & Econômicos \\
\hline Municípios & $\begin{array}{c}\text { Média do } \\
\text { município }\end{array}$ & Performance \\
\hline Bernardino Batista & $\mathbf{0 , 1 5}$ & Crítica \\
Cachoeira dos Índios & $\mathbf{0 , 1 7}$ & Crítica \\
Cajazeiras & $\mathbf{0 , 6 2}$ & Aceitável \\
Lastro & $\mathbf{0 , 2 5}$ & Crítica \\
Marizópolis & $\mathbf{0 , 0 5}$ & Crítica \\
Poço Dantas & $\mathbf{0 , 1 6}$ & Crítica \\
Poço de José de Moura & $\mathbf{0 , 1 9}$ & Crítica \\
Santa Cruz & $\mathbf{0 , 1 3}$ & Crítica \\
Santa Helena & $\mathbf{0 , 1 6}$ & Crítica \\
Santarém & $\mathbf{0 , 2 2}$ & Crítica \\
São Francisco & $\mathbf{0 , 1 9}$ & Crítica \\
São João do Rio do Peixe & $\mathbf{0 , 1 1}$ & Crítica \\
Sousa & $\mathbf{1 , 0 0}$ & Ideal \\
Triunfo & $\mathbf{0 , 0 5}$ & Crítica \\
Uiraúna & $\mathbf{0 , 1 2}$ & Crítica \\
Vieirópolis & $\mathbf{0 , 1 1}$ & Crítica \\
\hline Média dos municípios da sub-bacia & $\mathbf{0 , 2 3}$ & Crítica \\
\hline
\end{tabular}

Em relação à categoria abastecimento humano (tabela 3), o indicador foi constituído levando-se em consideração os parâmetros mostrados no Quadro 1. Percebe-se, conforme a tabela 3, que o desempenho dos indicadores dessa categoria se apresentou da seguinte forma: 19\% tiveram uma avaliação ideal (Sousa - 0,84, Marizópolis e Cajazeiras - 0,79 respectivamente); 44\% tiveram uma avaliação aceitável (Poço José de Moura - 0,67, Cachoeira dos Índios - 0,66, Triunfo-0,65, Uiraúna - 0,65, Santa Cruz - 0,65, Santa Helena - 0,58, Lastro - 0,51); 31\% tiveram uma avaliação de alerta (São Francisco - 0,48, São João do Rio do Peixe e Vieirópolis - 0,47, Santarém - 0,46 e Bernardino Batista -0,41); e 6\% tiveram uma avaliação crítica (Poço Dantas $0,23)$.

No geral a média dessa categoria foi igual a 0,58 demonstrando uma performance aceitável e que precisa de esforços na tentativa de melhorar este desempenho. 
Tabela 3 - Desempenho do Sistema quanto ao Abastecimento Humano

\begin{tabular}{lcc}
\hline Municípios & $\begin{array}{c}\text { Média do } \\
\text { município }\end{array}$ & Performance \\
\hline Bernardino Batista & $\mathbf{0 , 4 1}$ & Alerta \\
\hline Cachoeira dos Índios & $\mathbf{0 , 6 6}$ & Aceitável \\
Cajazeiras & $\mathbf{0 , 7 9}$ & Ideal \\
Lastro & $\mathbf{0 , 5 1}$ & Aceitável \\
Marizópolis & $\mathbf{0 , 7 9}$ & Ideal \\
Poço Dantas & $\mathbf{0 , 2 3}$ & Crítica \\
\hline Poço de José de Moura & $\mathbf{0 , 6 7}$ & Aceitável \\
Santa Cruz & $\mathbf{0 , 6 5}$ & Aceitável \\
Santa Helena & $\mathbf{0 , 5 8}$ & Aceitável \\
Santarém & $\mathbf{0 , 4 6}$ & Alerta \\
São Francisco & $\mathbf{0 , 4 8}$ & Alerta \\
São João do Rio do Peixe & $\mathbf{0 , 4 7}$ & Alerta \\
Sousa & $\mathbf{0 , 8 4}$ & Ideal \\
Triunfo & $\mathbf{0 , 6 5}$ & Aceitável \\
Uiraúna & $\mathbf{0 , 6 5}$ & Aceitável \\
Vieirópolis & $\mathbf{0 , 4 7}$ & Alerta \\
\hline Média dos municípios da sub-bacia & $\mathbf{0 , 5 8}$ & Aceitável \\
\hline
\end{tabular}

$O$ índice de sustentabilidade hidroambiental desta categoria (conforme os parâmetros definidos no quadro 1) foi de 0,78 , onde mostra uma situação ideal em $69 \%$ dos municípios, $19 \%$ em situações de alerta e 13\% em situação aceitável. Na tabela 4 é possível visualizar o panorama dos indicadores, com os respectivos índices de sustentabilidade hidroambiental para essa categoria.

Esse resultado advém do fato de que, nessas localidades, as atividades econômicas principais não estão relacionadas a estas pressões (pecuária, irrigação, abastecimento rural, aquicultura e lazer), o que automaticamente minimiza os impactos sobre os recursos hídricos. Observe que a situação de Sousa já se configura como uma situação de alerta posto que existe uma forte pressão da irrigação nas Várzeas do Perímetro Irrigado do Açude de São Gonçalo, bem como em São João do Rio do Peixe e Santarém nos quais o montante de outorgas é considerável.

Tabela 4 - Desempenho do sistema quanto às pressões da irrigação, pecuária, abastecimento rural, aquicultura e lazer

\begin{tabular}{lcc}
\hline Municípios & $\begin{array}{c}\text { Média do } \\
\text { município }\end{array}$ & Performance \\
\hline Bernardino Batista & 0,95 & Ideal \\
Cachoeira dos Índios & 0,78 & Ideal \\
Cajazeiras & 0,65 & Aceitável \\
Lastro & 0,97 & Ideal \\
Marizópolis & 1,00 & Ideal \\
Poço Dantas & 0,88 & Ideal \\
Poço de José de Moura & 0,83 & Ideal \\
Santa Cruz & 0,90 & Ideal \\
Santa Helena & 0,85 & Ideal
\end{tabular}




\begin{tabular}{|c|c|c|}
\hline Santarém & 0,41 & Alerta \\
\hline São Francisco & 0,95 & Ideal \\
\hline São João do Rio do Peixe & 0,38 & Alerta \\
\hline Sousa & 0,43 & Alerta \\
\hline Triunfo & 0,82 & Ideal \\
\hline Uiraúna & 0,74 & Aceitável \\
\hline Vieirópolis & 0,94 & Ideal \\
\hline Média dos municípios da sub-bacia & 0,78 & Ideal \\
\hline
\end{tabular}

Fonte: Elaboração própria a partir dos dados da pesquisa.

No que se refere à categoria relacionada ao desempenho do sistema de quanto à coleta de esgotos (tabela 5) verifica-se que a média geral deste indicador apresentou resultado aceitável, uma vez que seu índice de sustentabilidade hidroambiental foi de 0,60 . Cerca de $87,5 \%$ dos municípios apresentaram situação dentro dos parâmetros aceitáveis. Destaque ainda que o município de Uiraúna que apresentou uma performance ideal $(0,77)$ e Vieirópolis que apresenta uma situação de alerta $(0,44)$.

Como essas variáveis são tidas como elementos que exercem forte pressão hídrica e apresentam números abaixo dos ideais, esses indicadores demonstram a necessidade de definição de políticas dos gestores públicos dos municípios analisados, mesmo em um cenário aceitável, em busca de manter ou melhorar tais resultados, sendo necessário maior investimento nesse tipo de infraestrutura.

Tabela 5 - Desempenho do sistema quanto à coleta de esgotos

\begin{tabular}{lcc}
\hline Municípios & $\begin{array}{c}\text { Média do } \\
\text { município }\end{array}$ & Performance \\
\hline Bernardino Batista & 0,64 & Aceitável \\
Cachoeira dos Índios & 0,62 & Aceitável \\
Cajazeiras & 0,65 & Aceitável \\
Lastro & 0,52 & Aceitável \\
Marizópolis & 0,54 & Aceitável \\
Poço Dantas & 0,64 & Aceitável \\
Poço de José de Moura & 0,54 & Aceitável \\
Santa Cruz & 0,61 & Aceitável \\
Santa Helena & 0,52 & Aceitável \\
Santarém & 0,54 & Aceitável \\
São Francisco & 0,56 & Aceitável \\
São João do Rio do Peixe & 0,64 & Aceitável \\
Sousa & 0,67 & Aceitável \\
Triunfo & 0,52 & Aceitável \\
Uiraúna & 0,77 & Ideal \\
Vieirópolis & 0,44 & Alerta \\
\hline Média dos municípios da sub-bacia & $\mathbf{0 , 6 0}$ & Aceitável \\
\hline & Fonte: Elaboração própria a partir dos dados da pesquisa.
\end{tabular}

Em relação à categoria desempenho do sistema quanto ao atendimento de coleta de lixo (tabela 6) contatou-se a seguinte performance para seus indicadores: $44 \%$ tiveram uma avaliação 
aceitável, 56\% tiveram uma avaliação alerta. Os municípios com melhores performances foram: São Francisco $(0,62)$, Santa Helena $(0,57)$ e Cajazeiras $(0,55)$. Notadamente, os piores desempenhos foram obtidos pelos seguintes municípios: Lastro $(0,29)$, Marizópolis, Poço José de Moura e Vieirópolis (todos com indicador 0,35).

O saldo desta categoria não foi muito satisfatório, uma vez que o seu índice de sustentabilidade foi de 0,46 , um nível considerado em estado de alerta. Tal resultado demonstra que os municípios localizados nesta sub-bacia hidrográfica necessitam rever algumas alternativas para melhoria em termos de coleta de lixo.

\begin{tabular}{lcc} 
Tabela 6 - Desempenho do sistema quanto ao atendimento de coleta de lixo \\
\hline Municípios & $\begin{array}{c}\text { Média do } \\
\text { município }\end{array}$ & Performance \\
\hline Bernardino Batista & 0,41 & Alerta \\
Cachoeira dos Índios & 0,53 & Aceitável \\
Cajazeiras & 0,55 & Aceitável \\
\hline Lastro & 0,29 & Alerta \\
Marizópolis & 0,35 & Alerta \\
Poço Dantas & 0,36 & Alerta \\
Poço de José de Moura & 0,35 & Alerta \\
Santa Cruz & 0,53 & Aceitável \\
Santa Helena & 0,57 & Aceitável \\
Santarém & 0,51 & Aceitável \\
São Francisco & 0,62 & Aceitável \\
São João do Rio do Peixe & 0,49 & Alerta \\
Sousa & 0,52 & Aceitável \\
Triunfo & 0,47 & Alerta \\
Uiraúna & 0,49 & Alerta \\
Vieirópolis & 0,35 & Alerta \\
\hline Média dos municípios da sub-bacia & $\mathbf{0 , 4 6}$ & Alerta \\
\hline
\end{tabular}

No que se refere ao desempenho do sistema quanto ao estado qualitativo da água (tabela 7), foi possível observar que alguns municípios localizados nesta sub-bacia não dispunham de informações segundo o SNIS - Sistema Nacional de Informações sobre Saneamento (2008), motivo que fez com que no cálculo da média fossem excluídos tais municípios. Nesse sentido, os municípios que apresentaram melhores desempenhos foram: Cachoeira dos Índios $(0,91)$, Uiraúna $(0,87)$ e Santa Cruz $(0,85)$. No geral, os municípios apresentaram razoáveis desempenhos, visto que, a maioria obteve indicador acima de 0,60 , o que denota parâmetros razoáveis em relação à qualidade da água disponibilizada. 
Tabela 7 - Desempenho do sistema quanto ao estado qualitativo da água

\begin{tabular}{|c|c|c|}
\hline Municípios & $\begin{array}{l}\text { Média do } \\
\text { município }\end{array}$ & Performance \\
\hline Bernardino Batista & - & Sem dados \\
\hline Cachoeira dos Índios & 0,91 & Ideal \\
\hline Cajazeiras & 0,62 & Aceitável \\
\hline Lastro & 0,84 & Ideal \\
\hline Marizópolis & 0,60 & Aceitável \\
\hline Poço Dantas & - & Sem dados \\
\hline Poço de José de Moura & - & Sem dados \\
\hline Santa Cruz & 0,85 & Ideal \\
\hline Santa Helena & 0,70 & Ideal \\
\hline Santarém & - & Sem dados \\
\hline São Francisco & - & Sem dados \\
\hline São João do Rio do Peixe & 0,74 & Aceitável \\
\hline Sousa & - & Sem dados \\
\hline Triunfo & 0,36 & Alerta \\
\hline Uiraúna & 0,87 & Ideal \\
\hline Vieirópolis & - & Sem dados \\
\hline Média dos municípios da sub-bacia & 0,72 & Aceitável \\
\hline
\end{tabular}

No intuito de sintetizar os resultados obtidos com a análise, encontram-se explicitadas no gráfico 1 o desempenho das sub-bacias por categoria/dimensão e os respectivos índices de sustentabilidade hidroambiental. A categoria econômica $(0,23)$ e a categoria de coleta de lixo $(0,46)$ foram as que apresentaram situação mais desfavorável (crítica e alerta respectivamente). De outro modo as categorias/dimensões que apresentaram melhores desempenhos foram: pressões sobre os recursos hídricos $(0,78)$, estado qualitativo da água $(0,72)$ e coleta de esgotos $(0,60)$.

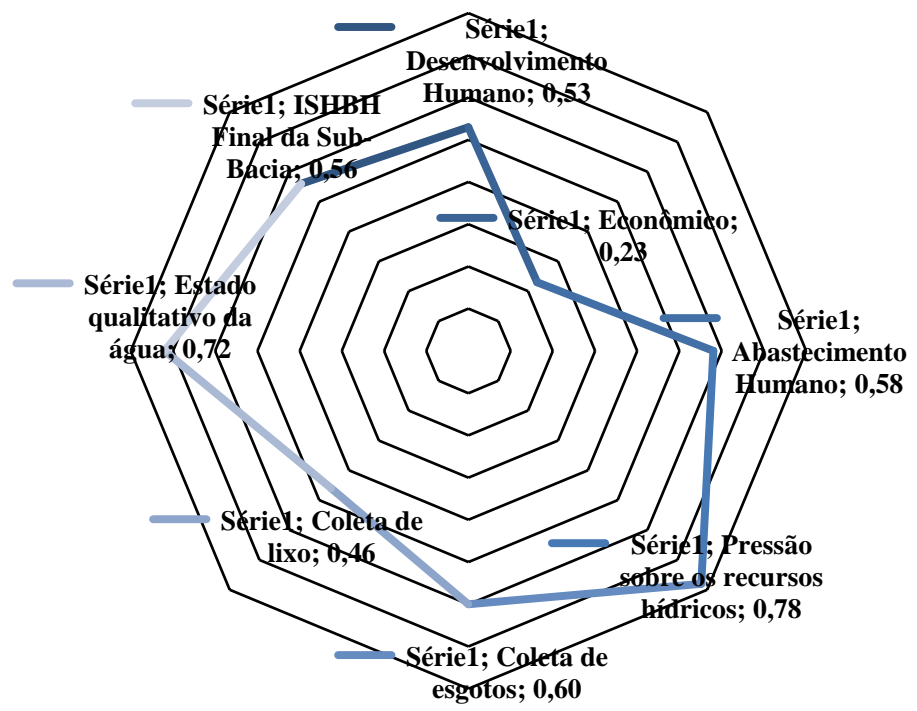

Gráfico 1 - Biograma geral das categorias/dimensões analisadas Fonte: Elaboração própria a partir dos dados da pesquisa. 
Finalmente, a partir do biograma apresentado no gráfico 2, é possível visualizar melhor os níveis de sustentabilidade hidroambiental dos municípios analisados. Os resultados obtidos apontam que os municípios com melhores performances hidroambiental foram: Cajazeiras $(0,67)$, Sousa $(0,60)$, Cachoeira dos Índios $(0,60)$, Uiraúna $(0,60)$, Santa Cruz $(0,59)$, Santa Helena $(0,57)$ e Lastro $(0,56)$. Os municípios com piores performance Poço Dantas $(0,36)$, Santarém $(0,38)$, Vieirópolis $(0,40)$ e Bernardino Batista $(0,43)$.

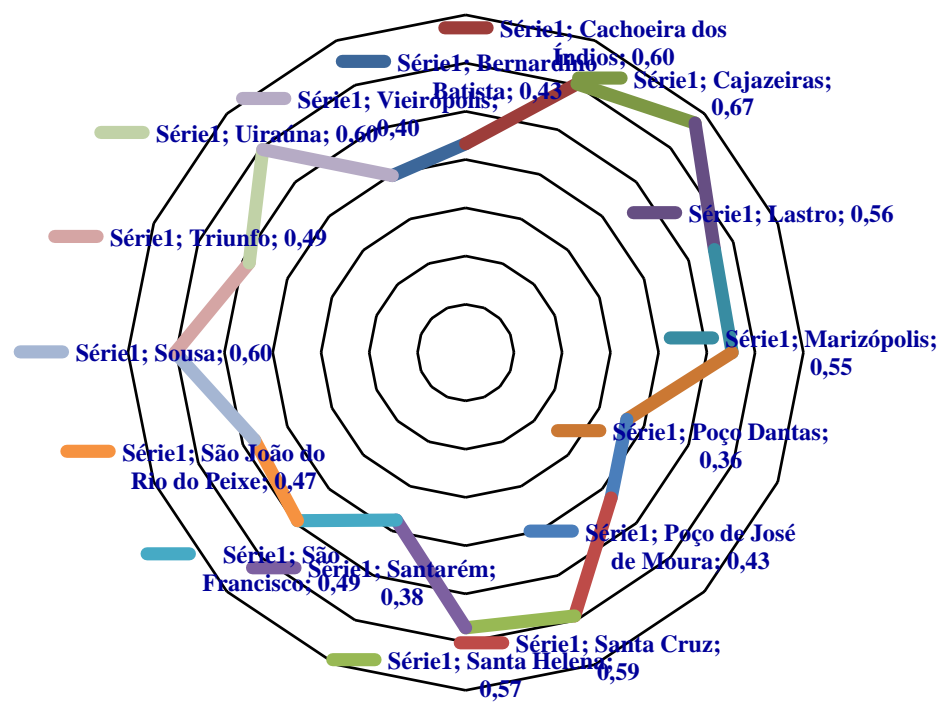

Gráfico 2 - Biograma do ISHBH dos Municípios da Sub-bacia Hidrográfica Fonte: Elaboração própria a partir dos dados da pesquisa.

Esses resultados encontrados através desse diagnóstico sinalizam a necessidade de melhorias relacionadas à gestão hidroambiental demonstrando a importância que deve ser dada por parte dos entes públicos (estadual e municipal) a esses municípios, introduzindo programas mais eficientes e eficazes para atender as demandas da gestão hídrica e ambiental, como por exemplo, ações mais "responsáveis" por parte das entidades reguladoras da gestão de águas no município paraibano de modo a proporcionar melhores desempenhos no futuro.

\section{CONSIDERAÇÕES FINAIS}

A partir dos resultados obtidos com a realização da presente pesquisa pode-se inferir que os aspectos relacionados às dimensões/categorias econômicas, coleta de lixo e desenvolvimento humano são aquelas que necessitam de mais ações, com vistas a melhorias nas questões relacionadas à educação, saúde e expectativa de vida, que direta ou indiretamente proporcionam entraves consideráveis relacionados à sustentabilidade hidroambiental da região estudada. Neste sentido, faz-se necessário buscar alternativas que possam potencializar o crescimento das questões destacadas, sendo essencial a integração e a inclusão dos diferentes atores sociais no processo, de forma que possa ser possível buscar alternativas capazes de reverter esse cenário.

Tal contexto e contingências levam à necessidade de serem definidas políticas públicas no sentido de estimular o crescimento da geração de alternativas sustentáveis de emprego e renda, dos níveis de educação ambiental, bem como em investimentos nas áreas diretamente relacionadas aos recursos hídricos, na tentativa de vislumbrar resultados mais satisfatórios. 
Finalmente, pode-se inferir e reconhecer, que o resultado referente à aplicação dessa metodologia para construção de um índice de sustentabilidade hidroambiental para bacias hidrográficas possibilita obter informações relevantes acerca da realidade estudada, através da mensuração de indicadores que forneceram informações para a identificação das dimensões/categorias analisadas. Todavia, e considerando os argumentos destacados por Cândido (2010), qualquer tentativa de avaliar o desenvolvimento e a sustentabilidade tem sempre muitas limitações e serão sempre passíveis de discussões, uma vez que se trata de questões complexas e paradoxais.

As reflexões, críticas e informações disponibilizadas neste trabalho se propõem a ampliar a discussão em torno das políticas públicas na área da gestão dos recursos hídricos, de modo a consubstanciar resultados mais satisfatórios a esse contexto, seja através do entendimento e ampliação da participação popular no processo de construção dessas políticas sociais, seja no aperfeiçoamento técnico dos órgãos gestores.

\section{REFERÊNCIAS}

1. ABERS, R.; JORGE, K. D. Descentralização da gestão da água: por que os comitês de bacia estão sendo criados? Revista Ambiente \& Sociedade, São Paulo, v.8, n. 2, p.99-124, jul./dez. 2005.

2. INSTITUTO DE DESENVOLVIMENTO MUNICIPAL E ESTADUAL. Anuário estatístico da Paraíba. João Pessoa: IDEME-PB, 2008. Disponível em: www.paraiba.pb.gov.br. Acesso em: 21 jan. 2011.

3. AGÊNCIA ESTADUAL DE ÁGUAS DA PARAÍBA - AESA. Mapas das bacias hidrográficas do Estado da Paraíba. Disponível em: http://www.aesa.pb.gov.br/geoprocessamento/geo portal/mapas.html. Acesso em: 29 fev. 2011.

4. CADERNO DE INFORMAÇÕES SOBRE SAÚDE PARAÍBA do Ministério da Saúde. Disponível em: http://tabnet.datasus.gov.br/tabdata/cadernos/pb.htm. Acesso em: 10 mar. 2011.

5. BRASIL. Sistema Nacional de Informações sobre Saneamento - SNIS. Diagnóstico dos serviços de água e esgotos, 2008. Disponível em: http://www.snis.gov.br/PaginaCarrega. php?EWRErterterTERTer=85. Acesso em: 15 mar. 2011.

6. BRASIL. Instituto Brasileiro de Geografia e Estatística. @Cidades. Disponível em: http://www.cidades.ibge.gov.br/xtras/home.php. Acesso em: 30 nov. 2011.

7. CÂNDIDO, G. A. Anotações de aula - Disciplina Sistema de Indicadores de Sustentabilidade. Programa de Pós-Graduação em Recursos Naturais (Doutorado) - PPGRN/UFCG, Campina Grande, PB, 2011.

8. CURI, R. C.; CURI, W. F.; OLIVEIRA, M. B.A. de. Análise de alterações na receita líquida otimizada de um perímetro irrigado no semiárido sob condições de variações hídricas e econômicas. Revista Brasileira de Recursos Hídricos, v. 9, n.3, p. 39-53, jul./set. 2004.

9. BECK, C. G.; ARAÚJO, A. C.; CÂNDIDO, G. A. Problemática dos resíduos sólidos urbanos do município de João Pessoa: aplicação do modelo P-E-R. Qualit@s Revista Eletrônica, Campina Grande, PB, v. 8, n. 3, p. 1-15, 2009.

10. BRAGA, T. M.; FREITAS, A. P. G. de; DUARTE, G. de S.; CAREPA-SOUZA, J. Índices de sustentabilidade municipal: o desafio de mensurar. Nova Economia. Setembro-dezembro. 
Belo Horizonte, MG, 2004.

11. KODIKARA, P.N.; PERERA, B. J. C.; KULARATHNA, M. D. U. P. Stakeholder preference elicitation and modelling in multi-criteria decision analysis - A case study on urban water supply. European Journal of Operational Research, v. 206, p.209-220, 2010.

12. GARCIAS, C. M. Indicadores de qualidade ambiental urbana. In: (Orgs.). N. B. Maia, H. L. Martos, \& W. Barrella. São Paulo: EDUC/COMPED/INEP, 2001.

13. GUIMARÃES, L. T. Proposta de um sistema de indicadores de desenvolvimento sustentável para bacias hidrográficas. 2008. 237 f. Tese (Doutorado em Ciências em Planejamento Energético) - Programa de Pós-graduação de Engenharia da Universidade Federal do Rio de Janeiro, Rio de Janeiro, RJ, 2008.

14. HE, C.; MALCOLM, S. B.; DAHLBERG, K. A.; FU, B. A conceptual framework for integrating hydrological and biological indicators into watershed management. Landscape and Urban Planning, 2000, pp. 25-34.

15. LAURA, A. A. Um método de modelagem de sistema de indicadores de sustentabilidade para a gestão dos recursos hídricos - MISGERH: O caso da bacia dos Sinos. 2004. 506 f. Tese (Doutorado em Recursos Hídricos e Saneamento Ambiental) - Programa de Pós-Graduação em Recursos Hídricos e Saneamento Ambiental. Universidade Federal do Rio Grande do Sul. Porto Alegre/RS, 2004.

16. LIRA, W. S. Sistema de gestão do conhecimento para indicadores de sustentabilidade SIGECIS: proposta de uma metodologia. 2008. $177 \mathrm{f}$. Tese (Doutorado em Recursos Naturais) - Universidade Federal de Campina Grande. Programa de Pós-Graduação em Recursos Naturais, Campina Grande, PB, 2008.

17. MAgAlHÃES JÚNIOR, A. P.; CORDEIRO NETTO, O. de M.; NASCIMENTO, N. de. O. Os Indicadores como instrumentos potenciais de gestão das águas no contexto legal-institucional do Brasil: resultado de um painel de especialistas. Revista Brasileira de Recursos Hídricos, v. 8, n. 9, p. 49-67, out./dez., 2003.

18. MAGALHÃES JÚNIOR, A. P. Indicadores ambientais e recursos hídricos: realidade e perspectivas para o Brasil a partir da experiência francesa. 2. ed. Editora Bertrand Brasil, 2010.

19. MARANHÃO, N. Sistema de indicadores para planejamento e gestão dos recursos hídricos de bacias hidrográficas. 2007. 397 f. Tese (Doutorado em Engenharia Civil) - Programa de PósGraduação em Engenharia Civil. Universidade Federal do Rio de Janeiro, Rio de Janeiro/RJ, 2007.

20. MARTINS, M. F., CÂNDIDO, G. A. Índice de desenvolvimento sustentável para municípios (IDSM): metodologia para análise e cálculo do IDSM e classificação dos níveis de sustentabilidade - uma aplicação no Estado da Paraíba. João Pessoa, PB: SEBRAE-PB, 2008.

21. MONTGOLFIER, J. de; NATALI, J. Instrumentos para uma Gestão Patrimonial. In: VIEIRA, P. F.; WEBER, J. (Orgs.). Gestão de recursos naturais renováveis e desenvolvimento: novos desafios para a pesquisa ambiental. Tradução Anne Sophie de Pontbriand Vieira, Christilla de Lassus. 3. ed. São Paulo: Cortez, 2002.

22. MUTIKANGA, H. E.; SHARMA, S. K.; VAIRAVAMOORTHY, K. Multi-criteria decision Analysis: A Strategic planning tool for water loss management. Water Resources Management, v. 25, p.3947-3969, 2011. 
23. POLICARPO, M. A.; SANTOS, C. R. dos. Proposta metodológica de uma gestão integrada e participativa dos recursos naturais de uso comum: a contribuição da análise Trade-Off. Revista de Estudos Ambientais, v.10, n. 2, p. 71-87, jul./dez., 2008.

24. POMPERMAYER, R. de S.; PAULA JÚNIOR, D. R. de; CORDEIRO NETTO, O. de M. Análise multicritério como instrumento de gestão de recursos hídricos: o caso das bacias dos rios Piracicaba, Capivari e Jundiaí. Revista Brasileira de Recursos Hídricos, vol. 12, no 3, p. 117127, jul/set, 2007.

25. SEPÚLVEDA, S. Desenvolvimento microrregional sustentável: métodos para planejamento local. Brasília: IICA, 2005.

26. SILVA, A. M. da; CORREIA, A. M. M., CÂNDIDO, G. A. Ecological footprint method: avaliação da sustentabilidade no município de João Pessoa, PB. In: G. A. Cândido (Org.). Desenvolvimento sustentável e sistemas de indicadores de sustentabilidade: formas de aplicações em contextos geográficos diversos e contingências específicas. Campina Grande, PB: Ed. UFCG, 2010.

27. ROOZBAHANI, A.; ZAHRAIE, B.; TABESH, M. PROMETHEE with precedence order in the criteria (PPOC) as a new group decision making AID: an application in urban water supply management. Water Resources Management, v. 26, p.3581-3599, 2012.

28. VAN BELLEN, H. M. Indicadores de sustentabilidade: uma análise comparativa. 2. ed. Rio de Janeiro: FGV, 2006.

29. VASCONCELOS, A. C. F. de; ANDRADE, E. de O.; MACEDO, N. M. M. N.; SILVA, R. M. da; CÂNDIDO, G. A. Análise da sustentabilidade entre municípios paraibanos do brejo paraibano: uma aplicação do índice de desenvolvimento sustentável municipal - IDSM. In: G. A. Cândido (Org.). Desenvolvimento sustentável e sistemas de indicadores de sustentabilidade: formas de aplicações em contextos geográficos diversos e contingências específicas. Campina Grande, PB: Ed. UFCG, 2010.

30. VIEIRA, P. M. S.; STUDART, T. M. C. Proposta metodológica para o desenvolvimento de um índice de sustentabilidade hidroambiental de áreas serranas no semiárido brasileiro - estudo de caso: Maciço de Baturité, Ceará. Revista Brasileira de Recursos Hídricos, vol.14, n. 4, p. 125-136, out./dez, 2009.

31. WAQUIL, P. D.; SCHNEIDER, S.; FILIPPI, E. E.; CONTERATO, M. A.; SPECHT, S. Para medir o desenvolvimento territorial rural: validação de uma proposta metodológica. In: XLV CONGRESSO DA SOCIEDADE BRASILEIRA DE ECONOMIA, ADMINISTRAÇÃO E SOCIOLOGIA RURAL, XLV, 2007, Londrina, PR. Anais... Londrina/PR: Universidade Estadual de Londrina, PR, 2007 